\title{
Geometric convexity of an operator mean
}

\author{
Shuhei Wada * \\ Department of Information and Computer Engineering, \\ National Institute of Technology, Kisarazu College
}

\begin{abstract}
Let $\sigma$ be an operator mean in the sense of Kubo and Ando. If the representation function $f_{\sigma}$ of $\sigma$ satisfies

$$
f_{\sigma}(t)^{p} \leq f_{\sigma}\left(t^{p}\right) \text { for all } p>1,
$$

then $\sigma$ is called a pmi mean. Our main interest is the class of pmi means (denoted by $P M I$ ). To study $P M I$, the operator mean $\sigma$, wherein

$$
f_{\sigma}(\sqrt{x y}) \leq \sqrt{f_{\sigma}(x) f_{\sigma}(y)} \quad(x, y>0)
$$

is considered in this paper. The set of such means (denoted by $G C V$ ) includes certain significant examples and is contained in $P M I$. The main result presented in this paper is that $G C V$ is a proper subset of $P M I$. In addition, we investigate certain operator-mean classes, which contain $P M I$.
\end{abstract}

\section{Introduction}

Let $\mathcal{H}$ be a Hilbert space with an inner product $\langle\cdot \mid \cdot\rangle$. A bounded linear operator $A$ on $\mathcal{H}$ is said to be positive (denoted by $A \geq 0$ ) if $\langle A x \mid x\rangle \geq 0$ for all $x \in \mathcal{H}$. We denote the set of positive operators on $\mathcal{H}$ by $B(\mathcal{H})_{+}$. If an operator $A \in B(\mathcal{H})_{+}$is invertible, we denote $A>0$.

A continuous real function $f$ from $(0, \infty)$ is said to be operator monotone on $(0, \infty)$, if the inequality $A \geq B>0$ implies $f(A) \geq f(B)$.

An operator monotone function $f$ on $(0, \infty)$ is called normal, if $f(1)=1$. In this paper, $O M_{+}^{1}$ denotes the set of normalized operator monotone functions on $(0, \infty)$ into itself.

In [7, Kubo and Ando provide the following axiom for operator means. A binary operation $\sigma$ among $B(\mathcal{H})_{+}$is called an operator mean, if it satisfies the following:

(i) $A \leq C, B \leq D \Rightarrow A \sigma B \leq C \sigma D$,

\footnotetext{
*E-mail: wada@j.kisarazu.ac.jp
} 
(ii) $T^{*}(A \sigma B) T \leq\left(T^{*} A T\right) \sigma\left(T^{*} B T\right)$,

(iii) $A_{n} \downarrow A, B_{n} \downarrow B \Rightarrow A_{n} \sigma B_{n} \downarrow A \sigma B$,

(iv) $1 \sigma 1=1$.

If $f$ is in $O M_{+}^{1}$, then the binary operation $\sigma_{f}$ on $B(\mathcal{H})_{+}$defined by

$$
A \sigma_{f} B=\lim _{\epsilon \downarrow 0} A_{\epsilon}^{\frac{1}{2}} f\left(A_{\epsilon}^{-\frac{1}{2}} B_{\epsilon}^{\frac{1}{2}} A_{\epsilon}^{-\frac{1}{2}}\right) A_{\epsilon}^{\frac{1}{2}}
$$

is an operator mean, where $A_{\epsilon}=A+\epsilon 1$ and $B_{\epsilon}=B+\epsilon 1$. Kubo and Ando show that the function $f \mapsto \sigma_{f}$ is an order isomorphism from $O M_{+}^{1}$ onto the set of operator means [7. In this paper, we call $\sigma_{f}$ an operator mean corresponding to $f$ and at times identify $\sigma_{f}$ as $f$.

The following theorem is referred to as the Ando-Hiai inequality ([2]). $A, B>$ $0, A \#_{\alpha} B \geq I \Rightarrow A^{p} \#_{\alpha} B^{p} \geq I \quad(p \geq 1)$, where $\alpha \in[0,1]$ and $\#_{\alpha}$ is an operator mean corresponding to a power function $t \mapsto t^{\alpha}$. In [14, it is shown that the generalized inequality

$$
A, B>0, A \sigma_{f} B \geq I \Rightarrow A^{p} \sigma_{f} B^{p} \geq I \quad(p \geq 1)
$$

holds if and only if $f$ is power monotone increasing (pmi for short), i.e.,

$$
f(t)^{p} \leq f\left(t^{p}\right) \quad(p \geq 1, t>0) .
$$

Our main interest is the class of pmi means (denoted by $P M I$ ). To study $P M I$, in this paper, we consider an operator mean $\sigma_{f}$, wherein

$$
f(\sqrt{x y}) \leq \sqrt{f(x) f(y)} \quad(x, y>0)
$$

holds. A positive valued function with 1.2 is called geometrically convex or multiplicatively convex ([3], 11]); hence, we denote the set of functions $f \in$ $O M_{+}^{1}$ with 1.2 by $G C V$.

In Section 3, we present some of the basic properties of $G C V$ and its adjoint (denoted by $G C C$ ). From this argument, we conclude that several significant $P M I$ means are contained in $G C V$.

It is conjectured that $G C V$ is a proper subset of $P M I$, i.e.,

$$
G C V \subsetneq P M I \text {. }
$$

In Section 4, we characterize a pmi mean and a gcv mean by using Hansen's integral representaion of an operator mean [5]. Using this, we prove the above conjecture, which is our main result.

In Section 5, we consider $P M I_{\infty}$ defined by

$$
P M I_{\infty}:=\left\{f \in O M_{+}^{1} \mid f(t) \geq t^{f^{\prime}(1)}\right\}
$$

and prove that $P M I$ is a proper subset of this class. 
Combining the above arguments, we finally obtain the following relationships: Let $\sigma$ be an operator mean and $f_{\sigma}$ be the representation function of $\sigma$. Consider the following statements:

(I) $f_{\sigma}$ is geometrically convex.

(II) $A, B>0, A \sigma B \geq I \Rightarrow A^{p} \sigma B^{p} \geq I \quad(p \geq 1)$.

(III) $\sigma \geq \#_{\alpha}$ for certain $\alpha \in[0,1]$. Then

(1) I implies II; II implies III,

(2) III does not imply II; II does not imply I.

\section{Geodesic mean}

As per the theory of Kubo and Ando [7, the set $O M_{+}^{1}$ of normalized positive operator monotone functions on $(0, \infty)$ is identified with the set of operator means. Hence, the following classes

$$
\mathcal{P} M I:=\left\{f \in O M_{+}^{1} \mid f(t)^{r} \leq f\left(t^{r}\right) \quad(\forall r>1)\right\}
$$

and

$$
\mathcal{P} M D:=\left\{f \in O M_{+}^{1} \mid f(t)^{r} \geq f\left(t^{r}\right) \quad(\forall r>1)\right\}
$$

can be viewed as subsets of the set of operator means. The function $f \in P M I$ (resp. $f \in P M D$ ) is referred to as a pmi (resp. pmd) mean. As stated in [14, for any probability measure $p$ on $[0,1]$, the function

$$
x \mapsto \int_{0}^{1} x^{\alpha} d p(\alpha)
$$

is in $P M I$. Such a function $f$ is called a geodesic mean and the set of geodesic means is denoted by $G M$. Several examples of a pmi mean can be obtained by using the fact that $G M \subset P M I$.

Although there are a number of functions belonging to PMI, it is not easy to show the pmi property (1.1) of a certain operator mean because the verification of condition (1.1) or (2.1) requires considerable calculation. Bourin and Hiai 3 mention that a positive operator monotone function $f$ on $[0, \infty)$ belongs to $G M$

if and only if $\frac{d^{n}}{d t^{n}} f\left(e^{t}\right) \geq 0$ for all $n \geq 0$. Thus we need to determine a technique for obtaining pmi means and evaluate this technique.

\section{Geometrically convex mean}

\subsection{Definitions and basic properties}

A positive function $f$ on $(0, \infty)$ is called geometrically convex (resp. geometrically concave), if

$$
f(\sqrt{x y}) \leq \sqrt{f(x) f(y)} \quad(\text { resp. } \quad f(\sqrt{x y}) \geq \sqrt{f(x) f(y)})
$$


holds for all $x, y>0$. Let $g c v$ (resp. $g c c$ ) be the set of monotone increasing continuous functions that are geometrically convex (resp. geometrically concave ) on $(0, \infty)$. We also define $G C V$ (resp. $G C C)$ as follows :

$$
G C V:=\left\{f \in O M_{+}^{1} \mid f \in g c v\right\} \quad\left(\text { resp. } G C C:=\left\{f \in O M_{+}^{1} \mid f \in g c c\right\}\right) .
$$

As stated in [3, the convexity of the function $t \mapsto \log f\left(e^{t}\right)$ is a necessary and sufficient condition for $f \in O M_{+}^{1}$ to be in $G C V$. Using this, we have some inclusions among the subclasses of $O M_{+}^{1}$. The second inclusion in the following is proved in 4 .

Proposition 3.1. $G M \subseteq G C V \subseteq P M I$.

Lemma 3.1. Let $f$ and $g$ be in gcv and let $h \in G C V$. Then we have the following.

(1) $f \cdot g$ and $f^{\alpha}$ are in $g c v$ for all $\alpha>0$;

(2) The function $t \mapsto\left(f \sigma_{h} g\right)(t)\left(:=f(t) \cdot h\left(\frac{g(t)}{f(t)}\right)\right)$ is in $g c v$;

(3) If $f$ is bijective, the inverse function of $f$ is in gcc.

Proof. The geometric convexity of $f \cdot g$ and $f^{\alpha}$ is immediate.

From the definition of $G C V$, inequalities

$$
\begin{aligned}
\left(f \sigma_{h} g\right)(\sqrt{x y}) & =f(\sqrt{x y}) \sigma_{h} g(\sqrt{x y}) \\
& \leq \sqrt{f(x) f(y)} \sigma_{h} \sqrt{g(x) g(y)} \\
& =\sqrt{f(x) f(y)} h\left(\sqrt{\left(\frac{g(x)}{f(x)}\right)\left(\frac{g(y)}{f(y)}\right)}\right) \\
& \leq \sqrt{f(x) f(y)} \sqrt{h\left(\frac{g(x)}{f(x)}\right) h\left(\frac{g(y)}{f(y)}\right)} \\
& =\sqrt{\left(f \sigma_{h} g\right)(x)\left(f \sigma_{h} g\right)(y)}
\end{aligned}
$$

hold for all $x, y>0$. This implies (2).

Let $f^{(-1)}$ be the inverse function of $f$. Then for every $x, y>0$,

$$
f\left(\sqrt{f^{(-1)}(x) f^{(-1)}(y)}\right) \leq \sqrt{x y}
$$

signifying that $f^{(-1)}$ is geometrically concave.

Using (2) of the above lemma, for $f, g \in g c v$, the weighted arithmetic mean of $f$ and $g$ is in $g c v$, which signifies that $g c v$ is a convex set. This implies that $G C V$ is a convex set. 
Proof of Proposition 3.1. From the above lemma, $G C V$ is a convex set and has a power function $x^{\alpha}(0 \leq \alpha \leq 1)$, which implies the first inclusion $G M \subseteq G C V$.

Next, we prove the second inclusion. As stated above, $f \in G C V$ if and only if function $t \mapsto F(t):=\log f\left(e^{t}\right)$ is convex on $(-\infty, \infty)$. Thus

$$
F((1-\alpha) t+\alpha s) \leq(1-\alpha) F(t)+\alpha F(s)
$$

holds for all $\alpha \in[0,1]$. Considering $t=0, F(\alpha s) \leq \alpha F(s)$, which implies that $f\left(x^{\alpha}\right) \leq f(x)^{\alpha}$ for all $x>0$.

Recall that $f(t) \mapsto f^{*}(t)\left(:=\frac{1}{f(1 / t)}\right)$ is an idempotent mapping on $O M_{+}^{1}$ and $P M D=P M I^{*}\left(:=\left\{f^{*} \mid f \in P M I I\right\}\right)$. The following is obtained.

Corollary 3.2.

$$
G M^{*} \subseteq G C C \subseteq P M D .
$$

Proof. From the preceding result,

$$
G M^{*} \subseteq G C V^{*}=G C C \subseteq P M I^{*}=P M D .
$$

Remark 3.1. From the above lemma, the set gcv is closed under the sum, i.e., $f_{1}, f_{2} \in g c v \Rightarrow f_{1}+f_{2} \in g c v$. However, the same does not hold for gcc. For example, $\frac{2 t}{t+1}$ and $t^{2}$ are in gcc and $\frac{2 t}{t+1}+t^{2}$ is not in $g c c$.

Before closing this section, we note that there are some counterexamples for $G M=G C V$.

Example 3.1. ([3]) Let $p \in[-1,1]$ and $b_{p}(t):=\left(\frac{t^{p}+1}{2}\right)^{1 / p}$. Then $b_{p} \in$ $G C V \backslash G M$ if and only if $p \in(0,1) \backslash\left\{\frac{1}{n} \mid n \in \mathbb{N}\right\}$.

Example 3.2. ([3]) Let $\alpha \in[-1,2]$ and $u_{\alpha}(t):=\frac{\alpha-1}{\alpha} \frac{t^{\alpha}-1}{t^{\alpha-1}-1}$. Then $u_{\alpha} \in$ $G C V \backslash G M$ if and only if $\alpha \in[1 / 2,2] \backslash\left\{1, \frac{m+1}{m}, \frac{m}{m+1} \mid m \in \mathbb{N}\right\}$.

\subsection{Functions in $G C V$}

In this section, we present a few examples of a function in $G C V(\subseteq P M I)$. We first consider the function $u_{\alpha}$ defined in the previous section. The geometric convexity of $u_{\alpha}$ is characterized as follows 3 :

$$
\left.\left.u_{\alpha} \in G C V \text { (resp. } u_{\alpha} \in G C C\right) \Longleftrightarrow 1 / 2 \leq \alpha \leq 2 \text { (resp. }-1 \leq \alpha \leq 1 / 2\right) .
$$

The function $u_{\alpha}$ is generalized as $u_{a, b}$ defined by

$$
u_{a, b}(t):=\frac{b}{a} \frac{t^{a}-1}{t^{b}-1} \quad(a, b \in[-2,2],(a, b) \neq(0,0)),
$$


where $\frac{t^{a}-1}{a}$ is defined as $\log t$, when $a=0$. In [9, Example 3.4(1)], it is proved that $u_{a, b} \in O M_{+}^{1}$ if and only if $(a, b)$ is in $\Gamma$, where

$$
\begin{aligned}
\Gamma:= & \{(a, b) \mid 0<a-b \leq 1,2 \geq a \geq-1,-2 \leq b \leq 1\} \\
& \cup([0,1] \times[-1,0]) \backslash\{(0,0)\} \\
& \cup\{(a, a) \mid a \neq 0\} .
\end{aligned}
$$

Proposition 3.3. $u_{a, b} \in G C V$ if and only if $(a, b) \in \Gamma$ and $|a| \geq|b|$.

Proof. We first consider the case, where $a b=0$. If $a=0$ and $b \neq 0$, then $u_{a, b}(t)=\frac{b}{t^{b}-1} \log t$ and

$$
\frac{d^{2}}{d x^{2}} \log u_{a, b}\left(e^{x}\right)=\frac{-1}{x^{2}}+b^{2}\left(e^{b x}+e^{-b x}-2\right)^{-1}<0
$$

for $x \neq 0$. Thus we obtain

$$
\frac{d^{2}}{d x^{2}} \log u_{b, a}\left(e^{x}\right)=-\frac{d^{2}}{d x^{2}} \log u_{a, b}\left(e^{x}\right) \geq 0,
$$

which implies the desired result.

We next consider the case, where $a \neq 0, b \neq 0$. Then there exists $\alpha \in \mathbb{R}$ such that

$$
u_{a, b}(t)=\frac{|b|}{|a|} \frac{t^{|a|}-1}{t^{|b|}-1} t^{\alpha}
$$

and

$$
\frac{d^{2}}{d x^{2}} \log u_{a, b}\left(e^{x}\right)=\frac{(|a| x)^{2} \psi(|a| x)-(|b| x)^{2} \psi(|b| x)}{x^{2}},
$$

where $\psi(x)=-\left(e^{x}+e^{-x}-2\right)^{-1}$. The function $\Psi(y):=y^{2} \psi(y)$ is a negative valued function on $(-\infty, \infty) \backslash\{0\}$ and

$$
\Psi(-y)=\Psi(y), \quad \Psi(x)<\Psi(y)
$$

for $0<x<y$. Thus

$$
\frac{d^{2}}{d x^{2}} \log u_{a, b}\left(e^{x}\right) \geq 0 \Longleftrightarrow \Psi(|a| x) \geq \Psi(|b| x) \text { for all } x \neq 0 \Longleftrightarrow|a| \geq|b| .
$$

From

$$
\frac{d^{2}}{d x^{2}} \log u_{a, b}^{*}\left(e^{x}\right)=\frac{d^{2}}{d x^{2}} \log u_{b, a}\left(e^{x}\right)=-\frac{d^{2}}{d x^{2}} \log u_{a, b}^{*}\left(e^{x}\right),
$$

the following is obtained.

Corollary 3.4. $u_{a, b} \in G C C$ if and only if $(a, b) \in \Gamma$ and $|a| \leq|b|$.

From the above results, we have $u_{a, b} \in G C V \cup G C C$ for all $(a, b) \in \Gamma$, which implies a condition for $u_{a, b}$ to be in $P M I$. 
Corollary 3.5. Let $(a, b) \in \Gamma$. Then the following are equivalent:

(1) $|a| \geq|b|($ resp. $|a| \leq|b|)$;

(2) $u_{a, b} \in G C V$ (resp. $u_{a, b} \in G C C$ );

(3) $u_{a, b} \in P M I$ (resp. $\left.u_{a, b} \in P M D\right)$.

The Stolarsky mean is defined as

$$
S_{\alpha}(s, t):=\left(\frac{s^{\alpha}-t^{\alpha}}{\alpha(s-t)}\right)^{\frac{1}{\alpha-1}} \text { for } \alpha \in[-2,2] \backslash\{0,1\},
$$

$S_{0}(s, t):=\lim _{\alpha \rightarrow 0} S_{\alpha}(s, t)$ and $S_{1}(s, t):=\lim _{\alpha \rightarrow 1} S_{\alpha}(s, t)$. It is known that $S_{\alpha}(1, t)$ is operator monotone, if $-2 \leq \alpha \leq 2$ [10. Using

$$
\log S_{\alpha}\left(1, e^{x}\right)=\frac{1}{\alpha-1} \log u_{\alpha, 1}\left(e^{x}\right),
$$

we have a condition for $S_{\alpha}(1, t)$ to be in $G C V$.

Corollary 3.6. $S_{\alpha}(1, t) \in G C V \quad$ (resp. $\left.S_{\alpha}(1, t) \in G C C\right)$ if and only if $\alpha \in$ $[-1,2]$ (resp. $\alpha \in[-2,-1]$ ).

Proof. By simple calculation, we have

$$
\frac{d^{2}}{d x^{2}} \log S_{1}\left(1, e^{x}\right)=\frac{d^{2}}{d x^{2}}\left(\frac{x e^{x}}{e^{x}-1}\right) \geq 0 .
$$

Thus $S_{1}(1, t) \in G C V$.

We next consider the case, where $\alpha \neq 1$. By Proposition 3.3

$$
\frac{d^{2}}{d x^{2}} \log u_{a, b}\left(e^{x}\right) \leq 0 \quad(\text { resp. } \geq 0) \Longleftrightarrow|a| \leq|b| \quad(\text { resp. }|a| \geq|b|) .
$$

Thus

$$
\begin{gathered}
\frac{d^{2}}{d x^{2}} \log S_{\alpha}\left(1, e^{x}\right)=\frac{1}{|1-\alpha|} \frac{d^{2}}{d x^{2}} \log u_{|\alpha|, 1}\left(e^{x}\right) \geq 0 \quad(1<\alpha \leq 2), \\
\frac{d^{2}}{d x^{2}} \log S_{\alpha}\left(1, e^{x}\right)=\frac{1}{|1-\alpha|} \frac{d^{2}}{d x^{2}} \log u_{1,|\alpha|}\left(e^{x}\right) \geq 0 \quad(-1 \leq \alpha<1)
\end{gathered}
$$

and

$$
\frac{d^{2}}{d x^{2}} \log S_{\alpha}\left(1, e^{x}\right)=\frac{1}{|1-\alpha|} \frac{d^{2}}{d x^{2}} \log u_{1,|\alpha|}\left(e^{x}\right) \leq 0 \quad(-2 \leq \alpha \leq-1) .
$$




\subsection{Inverses}

In [1, Ando proves that for every $f \in O M_{+}^{1}$, the function $t \mapsto t f(t)$ has the inverse function $(t f)^{(-1)}$ which is in $O M_{+}^{1}$, i.e.,

$$
f \in O M_{+}^{1} \Rightarrow(t f)^{(-1)} \in O M_{+}^{1} .
$$

In this section, we investigate this result with respect to the theory of geometrically convex functions.

Let $P$ be the set of nonnegative operator monotone functions on $[0, \infty)$ and

$$
P^{-1}:=\left\{h \in P \mid h([0, \infty))=[0, \infty), \quad h^{(-1)} \in P\right\} .
$$

In [13, Uchiyama proves the product formula

$$
P \cdot P^{-1} \subset P^{-1} \text {. }
$$

Using this, Ando's result stated above can be extended as

$$
f \in O M_{+}^{1} \Rightarrow\left(t^{\alpha} f\right)^{(-1)} \in O M_{+}^{1} \quad(\alpha \geq 1) .
$$

The following is immediate from the above argument.

Proposition 3.7. Let $\alpha \geq 1$ and $f \in O M_{+}^{1}$. Then

$$
f \in G C V \Longleftrightarrow\left(t^{\alpha} f\right)^{(-1)} \in G C C .
$$

Proof. Assume $f \in G C V$. Then it is evident that

$$
\frac{d^{2}}{d t^{2}} \log \left(t^{\alpha} f\right)\left(e^{t}\right)=\frac{d^{2}}{d t^{2}} \log f\left(e^{t}\right) \geq 0,
$$

which implies that $t^{\alpha} f \in g c v$ and $\left(t^{\alpha} f\right)^{(-1)} \in g c c$. The operator monotonicity of $\left(t^{\alpha} f\right)^{(-1)}$ comes from 3.1 .

Conversely, if $\left(t^{\alpha} f\right)^{(-1)} \in G C C,\left(t^{\alpha} f\right)$ is in $g c v$. Thus $\frac{d^{2}}{d t^{2}} \log f\left(e^{t}\right)=$ $\frac{d^{2}}{d t^{2}} \log \left(t^{\alpha} f\right)\left(e^{t}\right) \geq 0$.

From $\left(\left(t^{\alpha} f\right)^{(-1)}\right)^{*}=\left(t^{\alpha} f^{*}\right)^{(-1)}$ and $G C C^{*}=G C V$, the preceding proposition can be rewritten as follows:

Corollary 3.8. Let $\alpha \geq 1$ and $f \in O M_{+}^{1}$. Then

$$
f \in G C C \Longleftrightarrow\left(t^{\alpha} f\right)^{(-1)} \in G C V .
$$

We next consider a function $u(t)$ defined by

$$
u(t):=\beta \prod_{i=1}^{n}\left(t+a_{i}\right)^{\gamma_{i}},
$$

where $0=a_{1}<a_{2}<\cdots a_{n}, 1 \leq \gamma_{1}, 0<\gamma_{i}$ and $0<\beta$. Uchiyama shows that $u$ is in $P^{-1}$ and this result can be derived using the above product formula (12, 13]). Additionally, we show the following. 
Proposition 3.9. If $f \in G C V$ and $u(1)=1$, then $(u \cdot f)^{(-1)} \in G C C$.

Proof. From Lemma 3.1, we have $(u \cdot f) \in g c v$ and $(u \cdot f)^{(-1)} \in g c c$. The operator monotonicity of $(u \cdot f)^{(-1)}$ comes from 3.1 .

As the constant function 1 is in $G C V$, the following is evident:

Corollary 3.10. If $u(1)=1$, then $u^{(-1)} \in G C C$.

Example 3.3. For $\alpha \in(0,1)$, a function $u(t):=t(1-\alpha+\alpha t)$ has the inverse $u^{(-1)}(s)=\frac{\alpha-1+\sqrt{(1-\alpha)^{2}+4 s \alpha}}{2 \alpha}$ and $u^{(-1)}$ is in $G C C$.

\section{Main results}

In this section, we present an integral representation of an element of $G C V$. In [5], Hansen considers a class of real valued continuous functions defined as

$$
\mathcal{E}:=\left\{F \mid F: \mathbb{R} \rightarrow \mathbb{R} \text { is continuous and } e^{A} \leq e^{B} \Rightarrow e^{F(A)} \leq e^{F(B)}\right\},
$$

and proves the following :

(1) A function $F: \mathbb{R} \rightarrow \mathbb{R}$ is in $\mathcal{E}$ if and only if there exists $\beta \in \mathbb{R}$ and a measurable function, $h:(-\infty, 0] \rightarrow[0,1]$ such that

$$
F(x)=\beta+\int_{-\infty}^{0}\left(\frac{1}{\lambda-e^{x}}-\frac{\lambda}{\lambda^{2}+1}\right) h(\lambda) d \lambda,
$$

where $d \lambda$ is the Lebesgue measure on $(-\infty, 0]$;

(2) the preceding measurable function $h$ is uniquely determined by $F$;

(3) the function $F \mapsto \exp F(\log t)$ is a bijection from $\mathcal{E}$ onto $P$.

From this result, for $f \in O M_{+}^{1}, F(x)\left(:=\log f\left(e^{x}\right)\right)$ can be expressed as

$$
F(x)=\beta+\int_{-\infty}^{0}\left(\frac{1}{\lambda-e^{x}}-\frac{\lambda}{\lambda^{2}+1}\right) h(\lambda) d \lambda .
$$

As $F(0)=0$,

$$
\beta=\int_{-\infty}^{0}\left(\frac{-1}{\lambda-1}+\frac{\lambda}{\lambda^{2}+1}\right) h(\lambda) d \lambda
$$

Thus

$$
f(t)=\exp \int_{-\infty}^{0}\left(\frac{1}{\lambda-t}-\frac{1}{\lambda-1}\right) h(\lambda) d \lambda .
$$

Using this, we obtain the following : 
Proposition 4.1. Let $f \in O M_{+}^{1}$ and let $h$ be a measurable function determined using the above method. Then $f \in P M I$ if and only if

$$
\int_{-\infty}^{0}\left(\frac{1}{\lambda-t^{r}}-\frac{r}{\lambda-t}+\frac{r-1}{\lambda-1}\right) h(\lambda) d \lambda \geq 0
$$

for all $t>0$ and $r \geq 1$. Moreover, $f \in G C V$ if and only if

$$
\int_{-\infty}^{0}\left(\frac{\lambda+t}{(\lambda-t)^{3}}\right) h(\lambda) d \lambda \geq 0
$$

for all $t>0$.

Proof. From the above argument,

$$
F(x)=\log f\left(e^{x}\right)=\int_{-\infty}^{0}\left(\frac{1}{\lambda-e^{x}}-\frac{1}{\lambda-1}\right) h(\lambda) d \lambda .
$$

Hence, the condition $f\left(e^{r x}\right) / f\left(e^{x}\right)^{r} \geq 1$ can be expressed as

$$
0 \leq F(r x)-r F(x)=\int_{-\infty}^{0}\left(\frac{1}{\lambda-e^{r x}}-\frac{r}{\lambda-e^{x}}+\frac{r-1}{\lambda-1}\right) h(\lambda) d \lambda
$$

for all $x \in \mathbb{R}$ and $r \geq 1$.

From Lebesgue's dominated convergence theorem, the condition

$$
\frac{d^{2}}{d x^{2}} \log f\left(e^{x}\right) \geq 0
$$

can be expressed as

$$
\begin{aligned}
F^{\prime \prime}(x) & =\int_{-\infty}^{0} \frac{d^{2}}{d x^{2}}\left(\frac{1}{\lambda-e^{x}}-\frac{1}{\lambda-1}\right) h(\lambda) d \lambda \\
& =\int_{-\infty}^{0}\left(\frac{e^{x} \lambda+e^{2 x}}{\left(\lambda-e^{x}\right)^{3}}\right) h(\lambda) d \lambda \geq 0,
\end{aligned}
$$

which implies the desired result.

Remark 4.1. Let $0<a<\infty$. Considering $h=I_{(-\infty,-a)}\left(\right.$ resp. $\left.h=I_{(-a, 0)}\right)$, we have

$$
f(t)=\frac{a+t}{a+1} \in G C V \quad\left(\text { resp. } \quad f(t)=\frac{(a+1) t}{a+t} \in G C C\right) .
$$

Remark 4.2. Let $\alpha \in[0,1]$. Considering $h=\alpha I_{(-\infty, 0)}$,

$$
f(t)=t^{\alpha} \in G C V \cap G C C .
$$




\subsection{Conjecture and theorem}

It is conjectured that $G C V$ is a proper subset of $P M I$. To prove this, we use the argument of the preceding section. We set $\alpha=\frac{9}{14}$ and $h(\lambda):=\alpha I_{(-\infty,-2)}+$ $(1-\alpha) I_{(-1,0)}$ and show that inequality 4.2 does not hold, but 4.1) holds.

\section{Theorem 1.}

$$
G C V \subsetneq P M I .
$$

Proof. Let us show that 4.1) holds. For $r>1$, we have

$$
\begin{aligned}
\int_{-\infty}^{0} & \left(\frac{1}{\lambda-t^{r}}-\frac{r}{\lambda-t}+\frac{r-1}{\lambda-1}\right) h(\lambda) d \lambda \\
& =(1-\alpha)\left(\frac{\alpha}{1-\alpha} \log \frac{3^{r-1}\left(t^{r}+2\right)}{(t+2)^{r}}-\log \frac{2^{r-1}\left(t^{r}+1\right)}{(t+1)^{r}}\right) .
\end{aligned}
$$

Let us set $\beta:=\frac{\alpha}{1-\alpha}$ and

$$
\varphi(t):=\beta \log \frac{3^{r-1}\left(t^{r}+2\right)}{(t+2)^{r}}-\log \frac{2^{r-1}\left(t^{r}+1\right)}{(t+1)^{r}} .
$$

Then

$$
\frac{d \varphi}{d t}=\frac{2 r t\left(t^{r-1}-1\right) \psi_{\beta, r}(t)}{\left(t^{r}+2\right)\left(t^{r}+1\right)(t+2)(t+1)}
$$

where $\psi_{\beta, r}(t)=\left\{\left(\beta-\frac{1}{2}\right) t^{r+1}+(\beta-1)\left(t^{r}+t\right)-(2-\beta)\right\}$. Here, $\psi_{\beta, r}$ is strictly monotone increasing and equation $\psi_{\beta, r}(t)=0$ has a unique solution in $(0,1)$. Thus

$$
\min _{t \geq 0} \varphi(t)=\min \{\varphi(0), \varphi(1)\}=\varphi(1)=0 .
$$

We next show that inequality 4.2 does not hold. By simple calculation,

$$
\int_{-\infty}^{0}\left(\frac{\lambda+t}{(\lambda-t)^{3}}\right) h(\lambda) d \lambda=(1-\alpha) \frac{2}{(t+2)^{2}}\left(\beta-\frac{(t+2)^{2}}{2(t+1)^{2}}\right)
$$

where $\beta=\frac{\alpha}{1-\alpha}\left(=\frac{9}{5}\right)$. This takes a negative value, if $t$ is sufficiently small.

\section{Corollary 4.2.}

$$
G C C \subsetneq P M D
$$

\section{Related results}

In this section, we consider some operator-mean classes containing PMI and prove certain relationships among them. For $r>1$, we define

$$
P M I_{r}:=\left\{f \in O M_{+}^{1} \mid f\left(t^{r}\right) \geq f(t)^{r}\right\}
$$

and

$$
P M I_{\infty}:=\left\{f \in O M_{+}^{1} \mid f(t) \geq t^{\alpha} \text { for some } \alpha \in[0,1]\right\}
$$


We first note a property of $P M I_{r}$. It follows from [6, Corollary 4.7] that the Ando-Hiai type inequality,

$$
A, B>0, \quad A \sigma_{f} B \geq 1 \Rightarrow A^{r} \sigma_{f} B^{r} \geq 1
$$

is a necessary and sufficient condition for $f \in O M_{+}^{1}$ to be in $P M I_{r}$. In addition, from the proof of [14, Lemma 2.1], we have

$$
P M I=\bigcap_{x \in(1,2]} P M I_{x} \subset P M I_{r} .
$$

We next consider the case, where $r=\infty$. Let $f \in O M_{+}^{1}$ such that $f(t) \geq t^{\alpha}$. Then,

$$
\frac{f(t)-f(1)}{t-1} \geq \frac{t^{\alpha}-1}{t-1} \quad(t>1)
$$

and

$$
\frac{f(t)-f(1)}{t-1} \leq \frac{t^{\alpha}-1}{t-1} \quad(t<1),
$$

which implies that $f^{\prime}(1)=\alpha$. Thus the definition of $P M I_{\infty}$ can be rewritten as follows:

$$
P M I_{\infty}=\left\{f \in O M_{+}^{1} \mid f(t) \geq t^{f^{\prime}(1)}\right\} .
$$

As stated in [15, the following relationship among $P M I_{r}$ and $P M I_{\infty}$ is known.

Proposition 5.1. ([15]) For $r>1$,

$$
P M I_{r} \subset P M I_{\infty} .
$$

Proof. Let $f \in P M I_{r}$. It is evident from the definition that

$$
f\left(t^{s_{n}}\right)^{1 / s_{n}} \leq f(t)
$$

for $s_{n}:=1 / r^{n}$. Thus

$$
\lim _{n \rightarrow \infty} \exp \left(\log \left(f\left(t^{s_{n}}\right)^{1 / s_{n}}\right)\right)=\exp \left(\lim _{n \rightarrow \infty} \frac{\log \left(f\left(t^{s_{n}}\right)\right)}{s_{n}}\right)=t^{f^{\prime}(1)} \leq f(t) .
$$

From the above discussion, the problem whether $P M I$ is a proper subset of $P M I_{\infty}$ arises (cf. [15]). We provide an answer to this problem.

\section{Proposition 5.2.}

$$
\bigcup_{r>1} P M I_{r} \subsetneq P M I_{\infty}
$$


Proof. We show that

$$
f(t):=\frac{(1 / 3) t+(2 / 3) t^{1 / 3}}{(1 / 3)+(2 / 3) t^{1 / 3}}
$$

is in $P M I_{\infty} \backslash P M I_{r}$ for all $r>1$.

Let us show $f \in P M I_{\infty}$. As the operator monotonicity of $f$ comes from $[8$, it is sufficient to show $f(t) \geq t^{f^{\prime}(1)}$. Set $g(t):=f(t)-t^{1 / 3}$, then

$$
g^{\prime}(t)=\frac{t^{1 / 3}\left(t^{1 / 3}-1\right)\left(t^{1 / 3}+1\right)\left(2 t^{1 / 3}+1\right)\left(4 t^{1 / 3}-1\right)}{24 t^{2}+36 t^{\frac{5}{3}}+18 t^{\frac{4}{3}}+3 t}
$$

and $g(0)=g(1)=0$, which implies that $g(t) \geq 0$ and $f(t) \geq t^{1 / 3}$.

In addition, from

$$
\lim _{t \rightarrow 0} \frac{f\left(t^{r}\right)}{f(t)^{r}}=\lim _{t \rightarrow 0} \frac{\left((1 / 3) t^{r}+(2 / 3) t^{r / 3}\right)}{\left((1 / 3)+(2 / 3) t^{r / 3}\right)} \frac{\left((1 / 3)+(2 / 3) t^{1 / 3}\right)^{r}}{\left((1 / 3) t+(2 / 3) t^{1 / 3}\right)^{r}}=2^{1-r}<1,
$$

we have $f \notin P M I_{r}$.

Combining all the results stated above, we obtain the following:

Corollary 5.3. Let $\sigma$ be an operator mean and $f_{\sigma}$ be the representation function of $\sigma$. Consider the statements:

(I) $f_{\sigma}$ is geometrically convex.

(II) $A, B>0, A \sigma B \geq I \Rightarrow A^{r} \sigma B^{r} \geq I$ for all $r>1$.

(III) $A, B>0, A \sigma B \geq I \Rightarrow A^{r} \sigma B^{r} \geq I$ for some $r>1$.

Then

(IV) $\sigma \geq \#_{\alpha}$ for some $\alpha \in[0,1]$.

(1) I implies II; II implies III; III implies IV,

(2) IV does not imply III; II does not imply I.

Thus a problem arises.

Problem 1. Let $r>1$. Then, $P M I=P M I_{r}$ ?

\section{Acknowledgement}

We gratefully acknowledge the helpful discussions with F. Hiai on several points of this paper. 


\section{References}

[1] T. Ando, Comparison of norms $\||f(A)-f(B) \|| \mid$ and ||$|f(|A-B|)|\|$, Math. Z. 197 (1988), 403-409.

[2] T. Ando and F. Hiai , Log majorization and complementary GoldenThompson type inequality, Linear Algebra Appl. 197 (1994), 113-131.

[3] J.-C.Bourin and F. Hiai, Jensen and Minkowski inequalities for operator means and anti-norms, Linear Algebra Appl. 456 (2014), 22-53.

[4] J.I. Fujii and T. Yamazaki, Power monotonicity for a path of operator means, submitted to Scientiae Mathematicae Japonicae.

[5] F.Hansen, Selfadjoint means and operator monotone functions, Math. Ann. 256 (1981), no.1, 29-35.

[6] F. Hiai, Y. Seo and S. Wada Ando-Hiai type inequalities for multivariate operator means, to appear in Linear and Multilinear Algebra

[7] F. Kubo and T. Ando, Means of positive linear operators, Math. Ann. 246 (1980), 205-224.

[8] F. Kubo, N. Nakamura, K. Ohno and S. Wada, Barbour path of operator monotone functions, Far East J. Math. Sci. (FJMS) 57 (2)(2011), 181-192.

[9] M.Nagisa and S. Wada Operator monotonicity of some functions, Linear Algebra Appl. 486 (2015), 389-408.

[10] Y. Nakamura, Classes of operator monotone functions and Stieltjes functions, in : H. Dym, et al. (Eds.), The Gohberg Anniversary Collection, vol. II, Oper. Theory Adv. Appl., vol. 41, Birkhäuser, 1989, pp. 395-404.

[11] C.P. Niculescu and L-E. Persson, Convex functions and their applications. A contemporary approach, CMS Books in Mathematics/Ouvrages de Mathématiques de la SMC. Springer, Cham, 2018

[12] M. Uchiyama, Operator monotone functions which are defined implicitly and operator inequalities, J. Funct. Anal. 175 (2000), no. 2, 330-347.

[13] M. Uchiyama, A new majorization between functions, polynomials, and operator inequalities, J. Funct. Anal. 231 (2006), 221-244.

[14] S. Wada, Some ways of constructing Furuta-type inequalities, Linear Algebra Appl. 457 (2014), 276-286.

[15] T.Yamazaki, An integral representation of operator means via the power means and an application to the Ando-Hiai inequality, arXiv:1803.04630. 